\title{
Transmission Scheme for Asymmetric MIMO Two-way Relay with Channel Estimation Error
}

\author{
Jinhong Fan $^{1,2}$, Chaowei Yuan ${ }^{1}$ and Ke Xiao ${ }^{2}$ \\ ${ }^{1}$ College of Information and Communication Engineering, Beijing University of \\ Posts and Telecommunications, Beijing, China \\ ${ }^{2}$ College of Information Engineering, North China University of Technology, \\ Beijing, China \\ qfjh0922@163.com
}

\begin{abstract}
In this paper, we consider the transmission scheme for asymmetric decode-and-forward (DF) multi-input multi-output (MIMO) two-way relay system with channel estimation error. In order to transmit with asymmetric data rates from the relay to the receivers according to their individual link qualities, we propose a novel transmission scheme for the broadcast phase by employing a priori bit information. The proposed scheme employs multi-antenna and network coding at the relay. The idea is the weaker link receiver exploits a priori bit information in each transmit symbol, so that it only needs to decode on a subset of the transmit symbol constellation. Subject to the same bit error rate constraint, the weaker link receiver can decode at lower signal-to-noise ratio compared to the stronger link. The different signal labeling used for mapping bits to symbols at the relay cause different minimum Euclidean distance, so it is shown to be crucial for the performance at the receivers. Subsequently, we investigate the effect of the channel estimation error on the system performance. Simulations show that the proposed transmission scheme can be applied to practical scenarios with asymmetric channel qualities.
\end{abstract}

Keywords: Decode-and-forward, Multi-Input Multi-Output Two-way Relay, Network coding, Asymmetric Channel, Transmission scheme, Channel estimation error

\section{Introduction}

Recently the relay has been widely applied because it can improve the spectral efficiency, extend the coverage in cellular network. There are different types of relays such as decode-and-forward (DF), amplify and forward and compress and forward. In order to recover the spectral efficiency loss in half-duplex relaying systems, the twoway relaying protocol as a new technique is proposed and has drawn considerable attention, where two wireless stations exchange data via a wireless relay and there is no direct connection between the two stations [1]. Motivated by the great success of network coding and multi-input multi-output(MIMO) techniques, DF MIMO two-way relay system has received special attentions in recent years $[2,3]$. Network coding can be used at the relay, where the relay combines the data on the bit level using the XOR operation before modulation [4]. The relay does not split the power for transmitting the two sets of data to the two nodes at the same time. Multiple antenna technology can also be used in the two-way relaying network, which can improve the performance in the fading environments by means of spatial diversity and increase the data rate by using spatial multiplexing scheme [5]. It is shown in [6] that it is possible for the relay to transmit information rates equal to the individual link capacities simultaneously to the two receiving stations by using random coding approaches. In [2, 3] [6], it is assumed that the channels from the relay to the two stations are symmetric.

In the practical system, the channel qualities from the relay to the two stations are most asymmetric. How to transmit data, so that the data rates from the relay are not limited by the weaker link of the two stations, is an important problem. The authors in 
[7] presented an effective outage evaluation approach of the asymmetric two-way amplify-and-forward relaying. An asymmetric modulation scheme, with physical layer network coding, was presented in order to improve the transmission reliability in twoway relay system in [8]. And most of the known results assume that the two sources can remove their own signals successfully during the second time slot. However, such perfect self-interference cancellation is based on the assumption of perfect channel state information (CSI) conditions. In practice, it is difficult to obtain perfect CSI due to channel estimation error, which results in a degradation of system performance. As such, the authors in $[9,10]$ take the channel estimation error into consideration. To the best of the authors' knowledge, a unified study for the transmission schemes with imperfect CSI in asymmetric MIMO two-way relay has not been reported yet in the literature.

\section{System Model}

We consider the two-way DF relaying system in this paper where the two stations $S_{1}$ and $S_{2}$ exchange data via a half-duplex relay $R$ as shown in Figure 1 in two phases: the multiple-access (MAC) phase and the broadcast (BRC) phase. In the MAC phase, the two stations transmit their data to the relay and the relay decodes the received signal. The decoded data are combined at the relay and are retransmitted back to the two stations in the BRC phase. The two stations get the needed information by selfinterference elimination using the self-information. Network coding can mix data from different nodes in network and transmit signal by using wireless radio characteristics, which can improve throughout of wireless communication system. It has been applied in the two-way relaying system. Multiple antennas can improve performance in fading environment and has been applied in the two-way relaying system. The network coding and multiple antenna technology are applied in the system at the same time, which can improve throughout from network coding and spatial multiplexing and ensure more reliable transmission from spatial diversity.

Source nodes $S_{1}$ and $S_{2}$ each have an antenna and $R$ has $N_{\mathrm{R}}=2$ transceiver antennas. We assume that there is no direct connection between the stations $S_{1}$ and $S_{2}$. The network coding method and modulation mapping scheme of the relay are known to $S_{1}$ and $S_{2}$. They communicate through a relay node $R$ that is within the range of both $S_{1}$ and $S_{2}$. Since the signals originating at $S_{1}$ and $S_{2}$ are mutually independent from each other and $R$ has two transceiver antennas, the system can be seen as a $2 \times 2$ virtual MIMO system with spatial multiplexing scheme. In the case of the relay node $R$ transmitting and the source $S_{1}$ and $S_{2}$ receiving information, the system can be viewed as two $2 \times 1$ MISO (Multiple Input Single Output) systems with spatial diversity scheme.

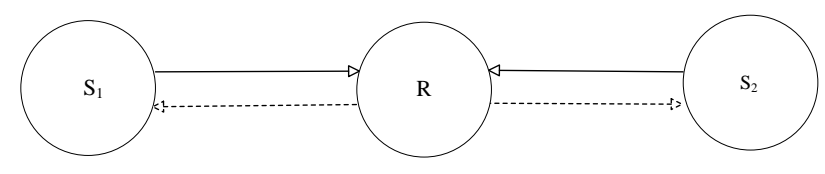

Figure 1. Two-Way Relaying System

In the MAC phase, the source nodes $S_{1}$ and $S_{2}$ transmit data simultaneously. The data sequence transmitted by the source nodes $S_{1}$ in the time slot $i$ is

$$
b_{i}^{1}=\left(b_{i, 1}^{(1)}, \ldots, b_{i, N}^{(1)}\right)^{T}
$$

$b_{i, n}^{(1)} \in\{0,1\}, n \in\{1, \ldots, N\}$, Let $b_{i}^{2}$ denote the data vector at source $S_{2}$ in the time slot $i$. The modulated signals of $S_{1}$ are denoted by $s_{i}^{(1)}(t)$ and $s_{i+1}^{(1)}(t)$ in the time slot $i$ and $(i+1) . s_{i}^{s_{i}^{(2)}(t)}$ 
And $s_{i+1}^{(2)}(t)$ denote the modulated signal of $S_{2}$ in the time slot $i$ and $(i+1)$. The relay node $R$ receives these signals via its two transceiver antennas. Let ${ }^{\left({ }_{i}(1)\right.}(t)$ denote the channel impulse response between the source nodes $S_{1}$ and the first antenna of the relay node $R$ in the time slot $i$. Let $h_{i}^{(1.2)}(t)$ denote the channel impulse response between the source nodes $S_{1}$ and the second antenna of the relay node $R$ in the time slot $i$. Similarly, ${ }_{i}^{(2.1)}(t)$ and $h_{i}^{(2.2)}(t)$ represent the channel impulse responses between the source node $S_{2}$ and the first as well as the second antenna of the relay node $R$. The channel impulse response approximately represents the single path channels with the negligible time variance. The general complex-valued numbers ${ }^{h_{i}^{(1.1)}}, h_{i}^{(1.2)}, h_{i}^{(2.1)}$ and $h_{i}^{(2.2)}$ can be used to represent the channel impulse responses. $n_{i}^{(1)}(t)$ and $n_{i}^{(2)}(t)$ denote the additive white Gaussian noise signals at the first and the second antenna of $R$ in the time slot $i$ and each having double-sided spectral noise power density $N_{0} / 2$, and with $e_{R, i}^{(1)}(t)$ and $e_{R, i}^{(2)}(t)$ being the received signals at the first and the second antenna of $R$ in the time slot $i$, the communication system is given by

$$
\left(\begin{array}{l}
e_{R, i}^{(1)}(t) \\
e_{R, i}^{(2)}(t)
\end{array}\right)=\left(\begin{array}{ll}
h_{i}^{(1,1)} & h_{i}^{(2,1)} \\
h_{i}^{(1,2)} & h_{i}^{(2,2)}
\end{array}\right)\left(\begin{array}{l}
s_{i}^{(1)}(t) \\
s_{i}^{(2)}(t)
\end{array}\right)+\left(\begin{array}{l}
n_{i}^{(1)}(t) \\
n_{i}^{(2)}(t)
\end{array}\right)
$$

The relay node $R$ determines the information contained in the received signals, yielding the detected versions $\tilde{b}_{i}^{1}=\left(\tilde{b}_{i, 1}^{(1)}, \ldots, \tilde{b}_{i, N}^{(1)}\right)$ and $\tilde{b}^{2}$.

In the BRC phase, the relay node $R$ re-encodes and re-modulates the received signals and forwards them to the source nodes. In this phase, network coding is exploited to get coding multiplexing gain. The received signal at the relay is denoted by $d_{i+2, n}^{(1)}$ and $d_{i+2, n}^{(2)}$

$$
\begin{aligned}
& d_{i+2, n}^{(1)}=\tilde{b}_{i, n}^{(1)} \oplus \tilde{b}_{i, n}^{(2)}, \quad d_{i+2, n}^{(1)} \in\{0,1\}, \quad n \in\{1, \ldots, N\} \\
& d_{i+2, n}^{(2)}=\tilde{b}_{i+1, n}^{(1)} \oplus \tilde{b}_{i+1, n}^{(2)}, \quad d_{i+2, n}^{(2)} \in\{0,1\}, \quad n \in\{1, \ldots, N\}
\end{aligned}
$$

$r_{i+2}^{(1)}(t), r_{i+2}^{(2)}(t), r_{i+3}^{(1)}(t)$ and $r_{i+3}^{(2)}(t)$ denote the modulated signal of the first antenna and second antenna at the relay $R$ in the time slot $(i+2)$ and $(i+3)$. After network coding, the ALAMOUTI scheme is applied with the formats $r_{i+3}^{(1)}(t)=-\left(r_{i+2}^{(2)}(t)\right) *$ and $r_{i+3}^{(2)}(t)=-\left(r_{i+2}^{(1)}(t)\right)^{*}$. The channel impulse response represent single path channels with negligible time variance, $h_{i+2}^{(1.1)}, h_{i+2}^{(1.2)}, h_{i+2}^{(2,1)}, h_{i+2}^{(2,2)}, h_{i+3}^{(i+1)}, h_{i+3}^{(1.2)}, h_{i+3}^{(2.1)}$ and $h_{i+3}^{(2,2)}$ denote the channel impulse response in the time slot $(i+2)$ and $(i+3)$. $n_{i+2}^{(i)}(t), n_{i+3}^{(1)}(t), n_{i+2}^{(2)}(t)$ and ${ }_{i+3}^{(2)}(t)$ denote the additive Gaussian white noise between the first antenna and second antenna of the relay $R$ and the source $S_{1}$. Thus the received signal $e^{(t)+2,2}(t)$ and $e^{e_{s+3}^{(t)}(t)}$ in the time slot $(i+2)$ and $(i+3)$ are

$$
\left(\begin{array}{l}
e_{s, i+2}^{(1)}(t) \\
e_{s, i+3}^{(1)}(t)
\end{array}\right)=\left(\begin{array}{ll}
h_{i+2}^{(1,1)} & h_{i+2}^{(1,2)} \\
\left(h_{i+3}^{(1,2)}\right)^{*} & -\left(h_{i+3}^{(1,1)}\right)^{*}
\end{array}\right)\left(\begin{array}{l}
r_{i+2}^{(1)}(t) \\
r_{i+2}^{(2)}(t)
\end{array}\right)+\left(\begin{array}{l}
n_{i+2}^{(1)}(t) \\
n_{i+3}^{(1)}(t)
\end{array}\right)
$$

Let $H_{R S 1}=\left(\begin{array}{ll}h_{i+2}^{(1,1)} & h_{i+2}^{(1,2)} \\ \left(h_{i+3}^{(1,2)}\right)^{*} & -\left(h_{i+3}^{(1,1)}\right)^{*}\end{array}\right)$.

And the received signal $e_{s+t, 2}^{(2)}(t)$ and ${ }^{e_{s, t s}^{(2)}(t)}$ are

$$
\left(\begin{array}{l}
e_{s, i+2}^{(2)}(t) \\
e_{s, i+3}^{(2)}(t)
\end{array}\right)=\left(\begin{array}{ll}
h_{i+2}^{(2,1)} & h_{i+2}^{(2,2)} \\
\left(h_{i+3}^{(2,2)}\right)^{*} & -\left(h_{i+3}^{(2,1)}\right)^{*}
\end{array}\right)\left(\begin{array}{l}
r_{i+2}^{(1)}(t) \\
r_{i+2}^{(2)}(t)
\end{array}\right)+\left(\begin{array}{l}
n_{i+2}^{(2)}(t) \\
n_{i+3}^{(2)}(t)
\end{array}\right)
$$

Let $H_{R S 2}=\left(\begin{array}{ll}h_{i+2}^{(2,1)} & h_{i+2}^{(2,2)} \\ \left(h_{i+3}^{(2,2)}\right)^{*} & -\left(h_{i+3}^{(2,1)}\right)^{*}\end{array}\right)$. 


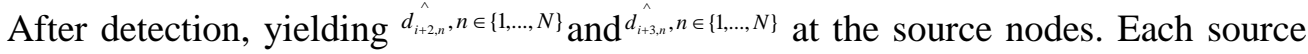
node is able to determine the particular information originated from the other source node. The source node $S_{1}$ computes

$$
\left(\begin{array}{l}
\hat{b}_{i+2, n}^{(1)} \\
b_{i+3, n}^{(1)}
\end{array}\right)=\left(\begin{array}{c}
\hat{d_{i+2, n}} \oplus b_{i, n}^{(1)} \\
\hat{d_{i+3, n}} \oplus b_{i+1, n}^{(1)}
\end{array}\right), n \in\{1, \ldots, N\}
$$

And the source nodes $S_{2}$ evaluates

$$
\left(\begin{array}{l}
\hat{b}_{i+2, n}^{(2)} \\
b_{i+3, n}^{(2)}
\end{array}\right)=\left(\begin{array}{l}
{\hat{d_{i+2, n}}}^{\wedge} \oplus b_{i, n}^{(2)} \\
d_{i+3, n} \oplus b_{i+1, n}^{(2)}
\end{array}\right), n \in\{1, \ldots, N\}
$$

In the following, we focus on the BRC phase and assume the MAC phase has been completed and the signal is correctly transmitted to $R$. Because the two channels from the relay to the source codes are asymmetric in practical scene, we can analysis the performance in the case. In order to meet the requirements of the bit error rate this paper uses the method of priori bit information considering the channel gaining of the weak link quality and strong link quality at the same time.

The perfect CSI is available at the receiver in above detection. However in practice the complex channel gain matrix $H$ has to be estimated at the receiver for retrieving the transmitted data symbol vector and imperfect channel estimation arises in any practical estimation scheme. Following the channel estimation model for MIMO systems in [8], we model the noisy channel estimate as

$$
\begin{aligned}
& \hat{H_{R S 1}}=H_{R S 1}+e_{1} \Omega_{1} \\
& \hat{H_{R S 2}}=H_{R S 2}+e_{2} \Omega_{2}
\end{aligned}
$$

Where $e_{1} \Omega_{1}$ is the estimation error that is uncorrelated with $H_{R S 1}, e_{2} \Omega_{2}$ is the estimation error that is uncorrelated with $H_{R S 2}$, the entries of $\Omega_{1}$ and $\Omega_{2}$ are i.i.d zero-mean complex Gaussian with unity variance and $e_{1}$ and $e_{2}$ are the measure of how accurate the channel estimation is.

\section{Transmission Scheme}

When the two channel qualities of the two-way relaying system are asymmetric for the BRC phase, a new method is proposed in order that the two channel gaining is each fully made use of. We assume that the signal is correctly transmitted to the relay node $R$ in the MAC phase.

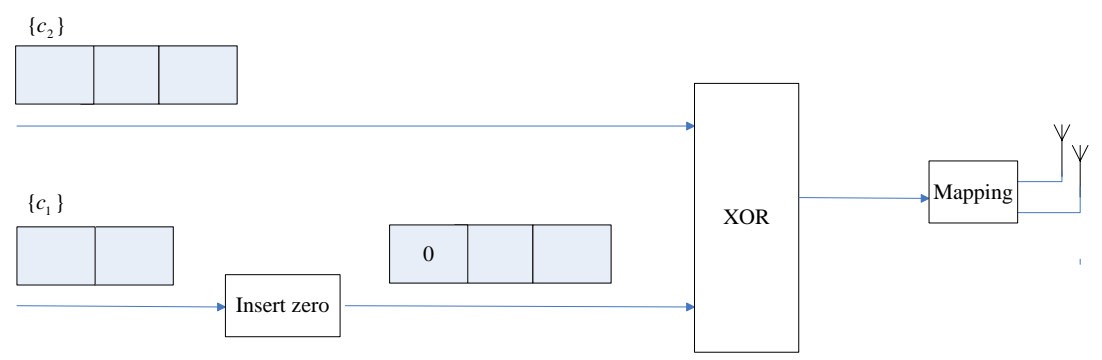

(a) Transmitter at $R$ 


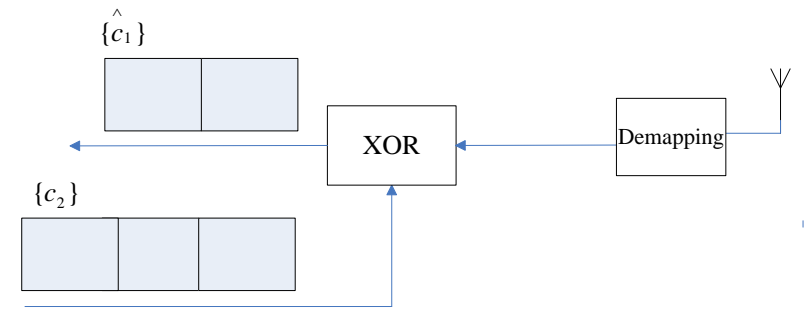

(b) Receiver at $S_{2}$

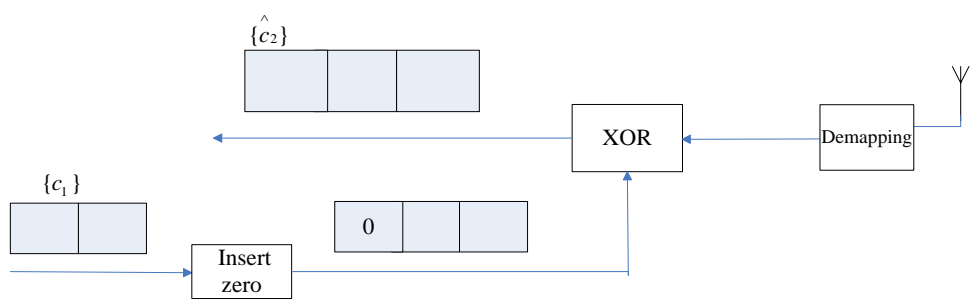

(c) Receiver at $S_{1}$

Figure 2. Transmitter and Receivers Diagrams in BRC Phase

In BRC phase, the transmitter $R$ and the receiver $S_{1}$ and $S_{2}$ diagrams of the proposed scheme are shown in Figure 2. The channels from the relay node $R$ to the two stations $S_{1}$ and $S_{2}$ are asymmetric. We assume that the link quality from $R$ to $S_{1}$ has the better channel quality, e.g., higher SNR, than the link from $R$ to $S_{2}$. The aim of the proposed scheme is to utilize the better link to transmit more data bits per channel use to the station $S_{1}$, at the same time the signal is transmitted to the station $S_{2}$ at a data rate that can be supported by its link. The proposed transmitter structure at the relay $R$ is shown in Figure 2(a). The transmitted signal of $S_{1}$ is $b^{b}$, which is partitioned into groups of $n N_{R}$ bits that is shown using $\left\{c_{1}\right\}$. The transmitted signal of $S_{2}$ is $b^{2}$, which is partitioned into groups of $m \mathrm{~N}_{\mathrm{R}}$ bits that is shown using $\left\{c_{2}\right\}$, and $n<m$. The value of $n$ and $m$ are decided by the received signal-to-noise ratio (SNR) of $S_{1}$ and $S_{2}$. We assume that ${ }^{b^{1}}$ and ${ }^{b^{2}}$ are correctly transmitted to $R$. The modulation method is $8 \mathrm{PSK}, M=2^{m}$. The $(m-n) \mathrm{N}_{\mathrm{R}}$ zeros is inserted into $c_{1}$, which is shown in $\tilde{c}_{1}=\left[0, \ldots 0, c_{1}^{n N_{R}}, \ldots, c_{1}^{1}\right]^{T}$. Those zeros contain no information. Their positions are fixed and known to both stations $S_{1}$ and $S_{2}$. After inserting zeros, the corresponding bits of $\bar{c}_{1}$ and $c_{2}$ are combined into ${ }^{c_{R}}$ using XOR operation.

$$
c_{R}=c_{2} \oplus \tilde{c_{1}}=\left[c_{2}^{m N_{R}}, \ldots, c_{2}^{n N_{R}+1}, c_{2}^{n N_{R}} \oplus c_{1}^{n N_{R}}, \ldots, c_{2}^{1} \oplus c_{1}^{1}\right]^{T}
$$

Due to the zeros in $\tilde{c}_{1}$, the ${ }^{\left[c_{2}^{m N_{R}}, \ldots, c_{2}^{n N_{R}+1}\right]}$ are kept unchanged after XOR operation when ${ }^{c_{R}}$ is generated, and those bits are known to the node $S_{2}$. The ${ }^{c_{R}}$ is modulated and the vector is $s_{R}=\left[s_{R, 1}, \ldots, s_{R, N_{R}}\right]^{T}=\mu\left(c_{R}\right)$, in which $\mu($.) is mapping function.

When the station $S_{1}$ receives signal, $c_{R}^{i}, \forall i \in\left\{1, \ldots, m N_{R}\right\}$ is useful information for the node $S_{1}$. In order to receive signal in certain bit error ratio (BER) in low $\mathrm{SNR}$, the priori bit information is used for the node $S_{2}$. Because $c_{R}^{i}, \forall i \in\left\{1, \ldots, n N_{R}\right\} \quad$ is useful information and $(m-n) N_{\mathrm{R}}$ bit information $\left[c_{2}^{m N_{R}}, \ldots, c_{2}^{n N_{R}+1}\right]$ is known to $S_{2}$, the subset of the mapping of the transmitted signal is demodulated. The subset is mapping that includes $\left[c_{2}^{m N_{R}}, \ldots, c_{2}^{n N_{R}+1}\right]$ in corresponding location. 


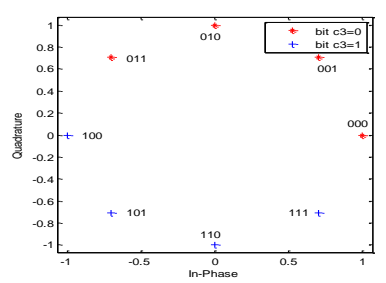

(a) Gray mapping

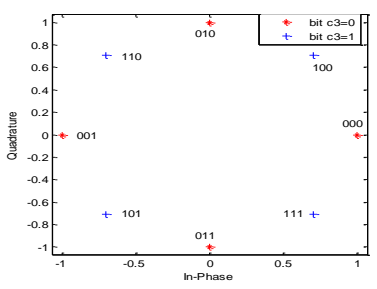

(b) set partition method 1

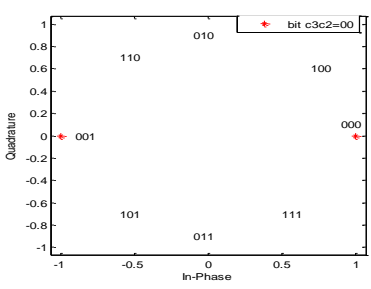

(c) set partition method 2

\section{Figure 3. 8PSK Mapping Method}

The 8PSK mapping method is shown in Figure 3. The gray mapping is shown in Figure 3(a). The set partition (SP) method 1 is shown in Figure 3(b). Assume that $n=2$ and $c_{2}^{3}=0$. The $c_{2}^{3}=0$ is known to the node $S_{2}$, the signal that the third bit is zero is only considered. Given the known bits $\left[c_{2}^{m N_{R}}, \ldots, c_{2}^{n N_{R}+1}\right]$, whose labels contain those known bits at the corresponding positions, as $s\left(c_{2}^{m N_{R}}, \ldots, c_{2}^{n N_{R}+1}\right) \subset \chi$

$$
s\left(c_{2}^{m N_{R}}, \ldots, c_{2}^{n N_{R}+1}\right)=\left\{s \mid c_{m N_{R}}(s)=c_{2}^{m N_{R}}, \ldots, c_{n N_{R}+1}(s)=c_{2}^{n N_{R}+1}, s \in \chi\right\}
$$

Where $c_{j}(s)$ denotes the $j$ th bit associated with label of the symbol $s$. Given $c_{2}^{3}=0$, the subset to be de-mapped at station $S_{2}$ can be denoted as $s(0)$. Figure 3(a) and 3(b) shows that different labeling schemes lead to different subsets for the third bit and affect the decoding performance at the receivers. For SP labeling, the components in $s(0)$ are same as those of QPSK and the minimum Euclidean distance between symbols in $s(0)$ is increased compared to that of the original 8PSK. However the minimum Euclidean distance of $s(0)$ for Gray labeling is not increased. The set partition mapping method 2 is shown in Figures 3(c), in which $n=2$ and $c_{2}^{3} c_{2}^{2}=00$, the mapped symbols are same as those of BPSK modulation.

\section{Performance Analysis}

In this section, we show the performance of the proposed asymmetric data rate transmission scheme and the effect of channel estimation error on the performance. The antennas of the relay $R$ is $N_{\mathrm{R}}=2$. The two channels from $R$ to $S_{1}$ and $R$ to $S_{2}$ are the Raleigh flat fading, but the two channel qualities are asymmetric. Noise is the Gaussian white noise with the mean value equal to 0 and the variance equal to 1 . The modulation mode is 8PSK. When $S_{1}$ and $S_{2}$ receive signal the minimum mean squared error (MMSE) detection is used [11, 12]. We assume that the target average BER for each user is $10^{-2}$. The information rate of $S_{1}$ is $m N_{\mathrm{R}}=6 \mathrm{bpt}$ and the information rate of $S_{2}$ is $n N_{\mathrm{R}}$. When $n=2, n N_{\mathrm{R}}$ is $4 \mathrm{bpt}$ and when $n=1, n N_{\mathrm{R}}$ is $2 \mathrm{bpt}$.

The scheme that Gray mapping is used at the relay $R$ is the traditional scheme. The scheme that SP labeling is used at the relay $R$ is the proposed scheme. The BER performance is shown in Figure 4. Since the transmissions to source nodes $S_{1}$ and $S_{2}$ do not interfere with each other, we show their performance in the same figures. The SNR on the $x$-axis represents $P_{R} / \sigma_{1}^{2}$ for transmission to the station $S_{1}$ and $P_{R} / \sigma_{2}^{2}$ for transmission to station $S_{2}$. The station $S_{2}$ can decode at lower SNR compared to the station $S_{1}$ by exploiting the a priori bit information subject to certain BER constraints. The comparison of the required SNR at BER $=10^{-2}$ is shown in Table 1 . When $\mathrm{BER}=10^{-2}$, the required SNR at $S_{2}$ of the $4 \mathrm{bpt}$ transmission rate is $1.2 \mathrm{~dB}$ lower than 
that of the station $S_{1}$ for the traditional scheme and the required SNR at source code $S_{2}$ of the $2 \mathrm{bpt}$ transmission rate is $3.1 \mathrm{~dB}$ lower than that of the station $S_{1}$ for the traditional scheme. The required SNR at the station $S_{2}$ of the $4 \mathrm{bpt}$ transmission rate is $7.5 \mathrm{~dB}$ lower than that of the station $S_{1}$ for the proposed scheme and the required SNR at the station $S_{2}$ of the $4 \mathrm{bpt}$ transmission rate is $16.2 \mathrm{~dB}$ lower than that of the station $S_{1}$ for the proposed scheme. The BER of the traditional scheme nearly equal because the Euclidean distances of different mapping schemes are same. The proposed scheme achieves coding gain of $4.3 \mathrm{~dB}$ to the traditional scheme for the station $S_{2}$ of the $4 \mathrm{bpt}$ transmission rate. Thus the stations $S_{1}$ and $S_{2}$ can receive data at the different transmission rate according to the different link quality. The channel gaining from $R$ to $S_{1}$ and $S_{2}$ is each made use of.

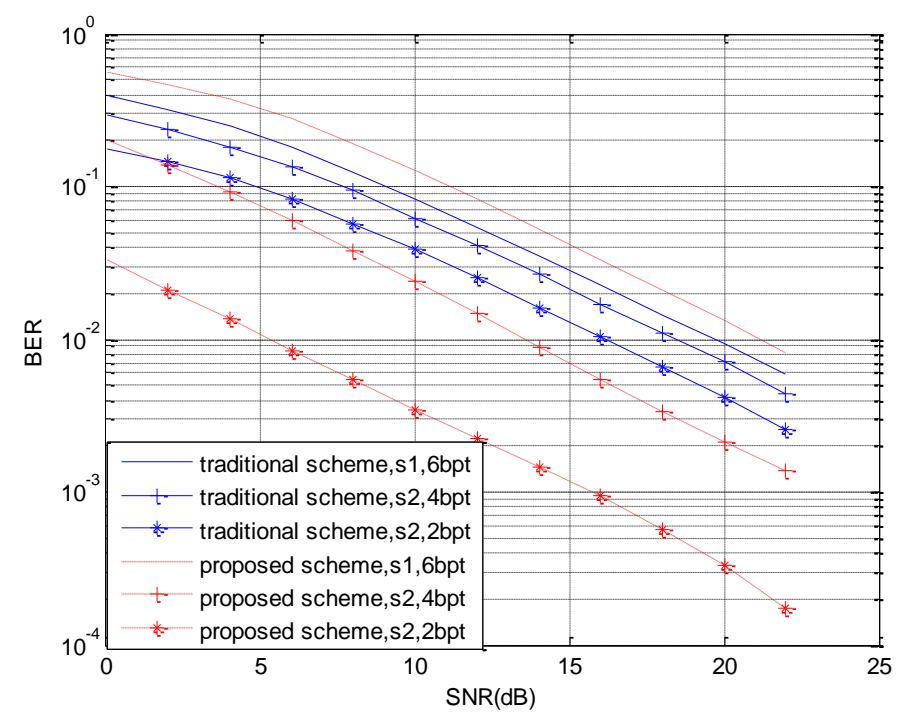

Figure 4. BER Performance

Table 1. Required SNR at BER $=10^{-2}$

\begin{tabular}{c|c|c|c}
\hline scheme & $S_{1}$ & $S_{2}, 4 \mathrm{bpt}$ & $S_{2}, 2 \mathrm{bpt}$ \\
\hline $\begin{array}{c}\text { Traditional } \\
\text { scheme }\end{array}$ & $\begin{array}{c}19.3 \\
\mathrm{~dB}\end{array}$ & $18.1 \mathrm{~dB}$ & $16.2 \mathrm{~dB}$ \\
$\begin{array}{c}\text { Proposed } \\
\text { scheme }\end{array}$ & 21.3 & $13.8 \mathrm{~dB}$ & $5.1 \mathrm{~dB}$ \\
$\mathrm{~dB}$ & & \\
\hline
\end{tabular}

In order to consider the effect of channel estimation error, BER performance in the presence of channel estimation error is investigated for $e_{1}=e_{2}=0.5,0.05$ in Figure 5 . From the figure we can see that BER performance is quite sensitive to channel estimation error and degrades significantly as $e_{1}$ and $e_{2}$ increased. 


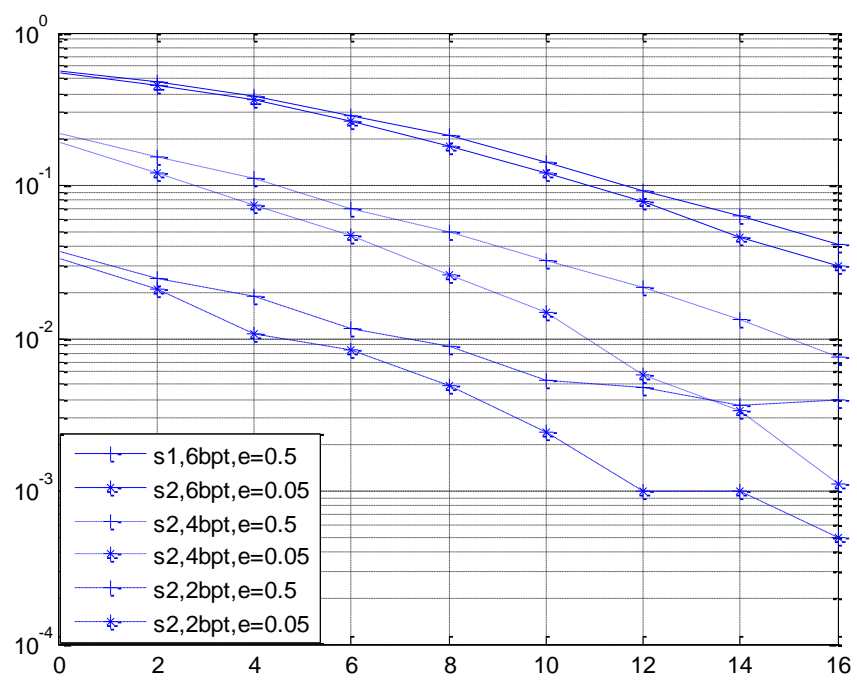

Figure 5. BER Performance of the Proposed Scheme with Channel Estimation Error

\section{Conclusions}

This paper addresses the transmission scheme for the asymmetric MIMO DF two-way relay system with channel estimation error. The idea is that the weaker link receiver exploits a priori bit information in each transmit symbol, so that it only needs to decode on a subset of the transmit symbol constellation. When the two link qualities are asymmetric, the weak link quality channel can attain to the same requirement of BER as the strong link quality. The simulation analysis show that in order to meet the requirement of BER the traditional method can adjust method only according to the weak link quality channel and the strong link quality channel gaining can not by fully made use of. In the proposed method, the data rates transmitted from the relay to the two receiving stations can be adjusted according to their individual link qualities subject to the certain BER constraints. And the channel estimation error is taken into consideration. When channel estimation error is bigger the BER performance decreases. The performance of the proposed method is obviously superior to the traditional method and it can be applied to the practical scene.

\section{Acknowledgements}

This work was supported in Scientific Research Common Program of Beijing Municipal Commission of Education under grant No.201310009002 and 2015 Scientific Research Common Program of Beijing Municipal Commission of Education.

\section{References}

[1] Rankov Boris, Wittneben Armin, "Spectral efficient protocols for half-duplex fading relay channels", IEEE Journal on Selected Areas in Communications, vol.25, no.2, pp.379-389, (2007).

[2] Baik Ihn Jung, Chung Sae Young, "Network coding for two way relay channels using lattices", IEEE ICC, pp.19-23,(2008).

[3] Xu Dong, Bai Zijin, Waadt Andreas, Bruck Guido, Jung Peter, "Combining MIMO with network coding: a viable means to provide multiplexing and diversity in wireless relay networks", IEEE ICC, pp.1-5, (2010). 
[4] Zhang Shengli, Liew Soung Chang, Lam Patrick P, "Hot Topic: physical layer network coding", Mobicom'06, pp.358-365, (2006).

[5] Alamouti Siavash M, "A simple transmit diversity technique for wireless communications", IEEE Journal on Selected Areas in Communications. Vol.16, no.8, pp.1451-1458, (1998).

[6] Oechtering Tobias J, Schnurr Clemens, Bjelakovic lgor, Boche Holger, "Broadcast capacity region of two phase bidirectional relaying", IEEE Transaction on Information Theory, vol.54, no.8, pp.454458, (2008).

[7] Jingjing Shi and Jianhua Ge, "General and efficient outage evaluation approach of asymmetric twoway AF relay", Electronics Letters, vol.49, no.25, pp.1645-1647, (2013).

[8] Wei Hao, Zheng Baoyu, and Ji Xiaodong, "A novel design of physical layer network coding in strong asymmetric two-way relay channels", Eurasip Journal on Wireless Communications and Networking, v2013, no.1, (2013).

[9] C.Wang, T.C.-K. Liu, and X.Dong, "Impact of channel estimation error on the performance of amplify and forward two way relaying”, IEEE Transaction on Vehicular Technology, vol.61, no.3, pp.1197-1207, (2012).

[10] Zhengyu Zhang, Jie Xu, and Ling Qiu, "Robust ARQ precoder optimization for AF MIMO relay systems with channel estimation errors", IEEE Transactions on Wireless Communications, vol.12, no.10, pp.5236-5247, (2013).

[11] Cheng Wang, "On the performance of the MIMO Zero-Forcing receiver in the presence of channel estimation error", IEEE Transaction on Wireless Communications, vol.6, no.3, pp.805-810, (2007).

[12] Kiessling M, Special J, "Analytical performance of MIMO MMSE receivers in correlated Raleigh fading environments", Vehicular Technology Conference, vol.3, no.8, pp.1738-1742, (2003). 
International Journal of Hybrid Information Technology

Vol.8, No.7 (2015) 